Revue d'histoire de l'Amérique française

Q REVUE D.HISTOIRE DE L'AMÉRIQUE FRANÇAISE

\title{
Descendances acadiennes
}

\section{Les quatre filles de Marie-Reine Berbudeau}

\section{Pierre Massé}

Volume 6, numéro 2, septembre 1952

URI : https://id.erudit.org/iderudit/301520ar

DOI : https://doi.org/10.7202/301520ar

Aller au sommaire du numéro

Éditeur(s)

Institut d'histoire de l'Amérique française

ISSN

0035-2357 (imprimé)

1492-1383 (numérique)

Découvrir la revue

Citer cet article

Massé, P. (1952). Descendances acadiennes : les quatre filles de Marie-Reine Berbudeau. Revue d'histoire de l'Amérique française, 6(2), 252-262.

https://doi.org/10.7202/301520ar d'utilisation que vous pouvez consulter en ligne.

https://apropos.erudit.org/fr/usagers/politique-dutilisation/ 


\section{DESCENDANCES ACADIENNES}

\section{LES QUATRE FILLES DE MARIE-REINE BERBUDEAU}

Sans doute, les documents sont rares qui permettent d'entrevo ir la nature des premiers rapports entre les réfugiés acadiens, à leur arrivée en Poitou, et les populations environnantes. Mais ils sont d'une grande précision. D'aspect revêche, ils ont peu tenté jusqu'à ce jour les chercheurs, qui vont plus volontiers aux épisodes pittoresques d'où l'on peut tirer des effets faciles. La réalité sociale du XVIIIe siècle finissant et du XIXe siècle à ses débuts repose dans ces recueils peu engageants que sont les actes d'État-Civil. Quiconque a la patience de dépouiller lentement les registres paroissiaux, de dresser des fiches, de suivre les filiations et les mariages, voit un jour apparaitre en pleine lumière l'explication de bien des faits.

Que des notaires compréhensifs consentent à ouvrir ensuite leurs minutes, et c'est une deuxième voie qui pénètre au centre de ce petit monde mal connu. Nous avons déjà appris avec curiosité que les paysans d'Archigny, jaloux des nouveaux venus et de leurs habitations toutes neuves, lancèrent des pierres sur les toitures et des pièces de bois dans les puits; que les habitants de Vicq et de Saint-Pierre-de-Maillé, réquisitionnés pour la construction des métairies, s'armèrent de fourches et de fusils contre la maréchaussée ${ }^{1}$. Événements colorés qui s'introduisent profondément au cœur du réel. Aussi riche d'enseignements est l'étude des alliances d'Acadiens et de Poitevins, se traduisant par la conquête ou la perte de la seule chose qui compte en ce pays sans industrie: la terre.

De la solidité ou de la fragilité de ces alliances, avec les facteurs susceptibles de les consolider ou désagréger, nous venons d'avoir un exemple avec la courte destinée de Françoise Texier, fille de

1. Ernest Martin, Les exilés acadiens en France au XVIII siècle et leur établissement en Poitou, (Paris, 1936), 215. 
Marie-Reine Berbudeau. Morte à 39 ans, laissant un mari criblé de dettes et trois enfants en bas âge dont on ne sait ce qu'ils sont devenus, le cas de cette infortunée est le type même de l'échec social. En regard de sa vie malencontreuse, les trois sœurs TexierLatouche restées à Bonneuil-Matours présentent une série réconfortante de prospérités.

L'Acadienne Marie-Reine Berbudeau était veuve depuis deux ans quand eut lieu, dans son quatuor de filles, le premier mariage. Marie-Anne devenait, le 16 juin 1806, la femme d'un jeune homme qui portait le même nom qu'elle: Bernard Texier.

Une fois de plus, nous constatons là une de ces unjons entre cousins dont on connait la fréquence dans les familles de bourgeoisie rurale, aux XVIIIe et XIXe siècles. Bernard Texier, le sergent forestier mort aveugle, le père du syndic des Acadiens, avait un neveu, Augustin Texier. C'est le fils de ce dernier, Bernard, qui épousa Marie-Anne. Il était donc, par rapport à sa jeune femme, un cousin issu de germain ${ }^{2}$.

Les origines d'Augustin Texier sont des plus modestes. Notre homme était un simple garçon huilier, en langage moderne: apprenti chez un patron, quand il épousa, le 8 novembre 1779, Jeanne Meunier fille d'un voiturier ${ }^{3}$. On appelait ainsi à l'époque et dans la région, les conducteurs qui menaient un attelage de bœufs pour le compte d'autrui ${ }^{4}$ Mi-journaliers, mi-laboureurs, et le plus souvent proletaires en mal d'argent, les charrois, effectués quelquefois à longue distance, leur apportaient quelque supplément de gain ${ }^{5}$. On peut croire que Jeanne Meunier et son mari furent un ménage où l'aisance matérielle ne régna pas souvent.

Après son mariage, Augustin Texier est devenu huilier, en 1780 , sans doute à son compte, en même temps que voiturier, comme

2. Pierre Massé, "Le syndic de la colonie acadienne en Poitou", Revue d'Histoire de l'Amérique française, janvier, septembre, décembre 1951. "Descendances acadiennes", mars 1952 .

3. Registres paroissiaux de Bonneuil-Matours. Toute date de naissance, mariage et décès est extraite de l'Etat Civil de cette commune. Dans nos articles précédents, nous avons indiqué l'origine des minutes de notaires utilisées.

4. Creuzé-Latouche, Description topographique du District de Châtellereault (Chatellereaut, 1790), 41.

5. Archives Nationales. $\mathrm{F}^{10} 212$; C.E. Labrousse, La Crise de l'Economie française à la fin de l'Ancien Régime et au début de la Révolution (Paris, 1944), 175. 
son beau-père. Mais c'est bientôt le départ en flèche lorsqu'il va s'établir cabaretier, à l'un des points cruciaux du bourg.

De ces endroits stratégiques pour la vie commerçante du XVIIIe siècle, Bonneuil-Matours en compte deux. Nous avons vu le syndic des Acadiens occuper le premier, à l'angle de la place de l'église où se presse, chaque dimanche, l'affluence du peuple et où siègent, avant la Révolution, les assemblées d'habitants chargées de gérer les affaires de la paroisse ${ }^{6}$. Augustin Texier s'est installé à l'autre bout de la Grand'Rue, en face de la porte du cimetière. Les vivants qui accompagnent les défunts n'ont qu'un pas à faire au cas où, à l'issue de la cérémonie, ils éprouveraient le besoin de se désaltérer. Derrière le champ du repos s'étend le champ de foire avec ses halles. Le lundi, jour de marché, est aussi jour faste pour le cabaretier, ainsi que les quatre foires annuelles, les plus fortes de la régjon ${ }^{7}$, amenant, de loin, une clientèle accrue.

Quelques faits significatifs jalonnent la montée sociale de l'aubergiste. Le 16 décembre 1780, Texier est encore un besogneux qui ne peut s'offrir le luxe d'affermer un carré de pré pour ses mules. Le fourrage atteint de hauts prix ${ }^{8}$ et les prairies naturelles se louent fort cher. C'est alors que le cabaretier jette ses regards sur le cimetière, de l'autre côté de la rue, où pousse une herbe abondante, et demande à l'abbé Gauvain de l'autoriser à couper cette provende. Il obtient aussi la liberté de défricher, épierrer et semer en luzerne une plate-bande de trois pieds tout le long des murs. Bail est dressé pour 9 ans, moyennant une ferme de 13 livres 5 sols au bénéfice de la paroisse. Mais, à l'expiration du bail, Texier n'aura plus besoin d'aller chercher son foin chez les morts. Car, bien vite, il a su se rendre utile, sinon indispensable, auprès d'une femme d'affaires

6. A. Babeau, Le village sous l'Ancien Régime (Paris, 1888), 63. Pour le Poitou, voir Prouhet, "Contribution à l'étude des Assemblées générales de communautés d'habitants sous l'Ancien Régime", Mémoires de la Societé des Antiquaires de l'Ouest (Poitiers, 1903) et A. Dernier, "Communauté d'habitants de Vouillé", Bulletin de la même société (1944), 473.

7. Archives Nationales. $F^{20}$ 391. Archives de la Vienne. L 314; Qn-1; L 336, fo 58: L 337, No 491; L 283, reg. 17; L 285, reg 19.

8. Gerbaux et Schmidt, Procès-verbaux des Comités d'agriculture et de commerce de la Constituante, de la Législative et de la Convention (Paris, 1906) I: 197. Octave Festy, L'Agriculture pendant la Revolution française. L'utilisation des jachères (Paris, 1950), 25 à 30. 
qui a des biens dispersés en Haut et Bas Poitou ${ }^{9}$ et a besoin d'un régisseur sérieux pour tenir la maison pendant son absence ${ }^{10}$. Elle a trouvé ce collaborateur en la personne d'Augustin Texier qui devient ainsi l'homme de confiance de madame de la Tousche, en attendant de devenir son homme de paille sous la Révolution.

C'est lui, en effet, qui va louer, en son propre nom, le 30 aout 1792, la métairie du Charraud, dans la commune voisine de Bellefonds, lorsque cette propriété sera mise sous séquestre et affermée comme bien national ${ }^{12}$. Il veilla sur l'immeuble pendant le cours du bail et put ainsi le restituer à sa propriétaire quand l'orage fut éloigné. Peut-on supposer que sa fidélité ne resta pas sans récompense? Et qu'on lui sent déjà les reins solides, puisqu'à la même époque, et par deux fois, on le choisit pour caution? C'est d'abord Touchois, administrateur du District, qui a jeté son dévolu sur une métairie d'émigré ${ }^{13}$. C'est ensuite Jean Penot et Jean Clerté, de Bonneuil-Matours désireux de se faire adjuger le vaste jardin d'un ci-devant ${ }^{14}$. Les uns et les autres s'arc-boutent sur la garantie d'Augustin Texier.

A l'ascension économique de l'ex-garçon huilier, en route vers la bourgeoisie, correspond une ascension politique non moins rapide. Membre de la municipalité en l'an II, et chargé de mettre en marche l'atelier révolutionnaire de salpêtre ${ }^{15}$, Texier figure parmi les notables communaux lorsque la constitution élaborée par Bonaparte met le point final à la Révolution ${ }^{16}$. On se souvient qu'aux élections de l'an XI pour le collège électoral de l'arrondissement de Châtellerault, c'est Texier-Latouche, syndic des Acadiens, qui avait obtenu le plus de voix: 109 suffrages sur 166 votants. Immédiatement après lui vient son cousin Augustin Texier, avec 96 voix $^{17}$. Consta-

9. Archives Nationales. $F^{7}$ 5776. Archives de la Vienne. $Q^{1} 44$.

10. Archives de la Vienne. L. 43.

11. Id. $\mathrm{L}^{5} 395$.

12. Ibid., L 447; L 337-56, fo 26 .

13. Ibid., $\mathrm{Q}^{1} 147$.

14. Reg. de délibérations de la municipalité de Bonneuil-Matours.

15. Archives de la Vienne. $\mathrm{E}^{4} 16$ bis-64.

16. Archives Nationales F1c III Vienne 1.

17. Ibid., F Ic III Vienne 2. 
tons que la boutique du premier, devant la place de l'église, et l'auberge du second, face au champ de foire et au cimetière, occupaient des emplacements de premier choix.

Maintenant, l'Empire est là. L'été de 1806 a ramené la paix. On peut entrevoir un avenir stable d'où les guerres seront bannies pour longtemps. Les deux fils de madame de la Tousche sont revenus d'émigration ${ }^{18}$, et déjà leur mère projette d'agrandir de quelques vignes la métairie retrouvée du Charraud ${ }^{19}$. Associé à Vincent Perrin, meunier, Texier a fait l'acquisition de la Talbatière, commune d'Archigny. Il possède désormais un pré, bien à lui, avec de l'herbe qu'un métayer lui fauche et conduit à domicile ${ }^{20}$. Le moment est venu de marier son fils aîné.

Ce fils, Augustin Texier n'avait jamais envisagé d'en faire un soldat. Le prestige de l'uniforme laissait insensible notre marchand qui, le 14 brumaire an XII, réunit dans son auberge un aréopage de onze villageois pour examiner le cas du jeune homme, dont les 21 ans allaient être touchés par la conscription. Le notaire Drouault, de Vouneuil-sur-Vienne, rédigea, d'une seule coulée, sur le papier timbré, cette formule que chaque membre de l'assemblée orna de sa signature:

"Onze habitants de Bonneuil-Matours certifient que Bernard Texier, conscrit de l'an XI, est atteint d'un rhumatisme à la cuisse et jambe gauche dont ils l'ont toujours connu boiteux; principalement depuis 4 ou 5 ans, époque où son mal a considérablement augmenté, et d'une telle manière que les comparants estiment qu'il est de justice de regarder et déclarer le dit Bernard Texier comme incapable de tout service militaire. ${ }^{21}$ "

On pouvait faire confiance au cabaretier pour utiliser convenablement le certificat. Il s'en servit de telle sorte que Bernard n'eut point à s'initier au métier des armes, et put convoler en justes noces

18. Archives de la Vienne. L 21.

19. Minutes Drouault.

20. Minutes Vézien.

21. Minutes Drouault. Sur l'attitude des populations de l'Ouest devant la conscription, voir G. Vallée, La conscription dans le département de la Charente (17981807), (Paris, 1937). 
avec la fille de l'Acadienne Marie-Reine Berbudeau, quelques années plus tard.

Le contrat de mariage fut signé le 9 juin 1806. La jeune MarieAnne recevait une dot de 935 livres, payable partie en nature. Une armoire à deux battants, un buffet, un lit garni, deux nappes, douze serviettes, deux chenets, deux petites tables, seraient, au départ, les meubles de la communauté. Mais le cadeau le plus important que l'Acadienne faisait au jeune ménage consistait dans "tous ustensiles servant à la boutique des marchands détaillants". Autrement dit, elle passait le fonds de commerce à sa fille et à son futur gendre qui habiteraient ainsi la maison venant du syndic des Acadiens, tandis que, vivant avec eux, elle se retirait des affaires. Le tout estimé à la valeur du moment, il restait 585 livres en argent effectif qu'elle verserait en plusieurs termes, le dernier à Noël 1808. En regard, Augustin Texier donnait à son fils 600 livres en numéraire. Les futurs époux ne seraient pas dans le besoin ${ }^{22}$.

La semaine d'après, jour pour jour, Bernard Texier épousait Marie-Anne Texier, sa cousine au deuxième degré. La profession que lui donne l'État-Civil indique avec éloquence la voie dans laquelle il s'engage, à 24 ans. On l'appelle fermier, c'est-à-dire homme d'affaires, marchand de biens comme nous écririons aujourd'hui. Il ne sera plus question pour lui de tirer de l'huile ou d'aller couper l'herbe du cimetière. Le fils du cabaretier et la fille de l'Acadienne fondent un foyer qui sera un foyer bourgeois.

Malheureusement, il y a cette inquiétude latente des guerres qui ne cessent point et deviennent vite de grandes mangeuses d'hommes. Il faudra renouveler bientôt l'exemption du service militaire. Devenu conscrit de 1808 comme il l'avait été de l'an XI, Bernard Texier entendra retentir encore l'impérieux appel aux armes. Mais son père est toujours là pour l'aider. Le 27 mars 1809, Augustin Texier conclut un traité a vec René Bourgueil, journalier de Vouneuil-sur-Vienne, qui payera l'impôt du sang à la place du jeune bourgeois, car il n'est plus question d'évoquer les rhumatismes. Il en coûtera cher à l'aubergiste: 2000 francs pour éloigner le danger ${ }^{23}$.

22. Minutes Drouault.

23. Minutes Collet. 
Nouvelle alerte en 1814. Depuis deux ans, l'Acadienne est morte. Ses deux filles plus jeunes sont mariées, nous verrons comment, et Bernard Texier occupe maintenant toute la maison, sur la place de l'église. Il doit faire, dans la Garde Nationale, un service de six mois qui ne l'enthousiasme pas plus que les précédents. Il s'en tire sans trop de frais, en se faisant remplacer par un cordonnier, Jacques Giraud, moyennant 400 francs seulement ${ }^{24}$. De l'Empire, Bernard Texier pourra dire ce que d'autres ont dit de la Terreur: j'ai vécu.

Trois enfants lui sont nés: Marie Anne, le 1er mai 1807; Marie Amélie, le 12 février 1809, et Pierre Augustin, le 20 mai 1811, décédé le 12 décembre suivant. A la Restauration, il n'a que 34 ans et profite de la conjoncture pour faire un nouveau bond en avant.

Faut-il s'étonner de le voir se transporter sur la colonie acadienne? Quand furent partagées, à la mort de Marie-Reine Berbudeau, les terres rassemblées par son mari, chacune des quatre filles eut son lot propre dont nous ignorons malheureusement la composition, l'acte notarié qui régla les modalités du partage nous étant inconnu. Nous avons dit, dans un article précédent, qu'à Françoise Reine, l'aînée, échut le hameau Saint-Antoine, composé des Nos 17 et 18. La présence de Bernard Texier dans la région, en 1819, nous incite à penser qu'il y avait également recueilli quelque bien.

L'affaire dont il va s'occuper est de celles qui doivent grossir ses revenus. Elle est aussi de celles qui mettent en lumière le jeu compliqué des transactions nouées et dénouées autour des métairies acadiennes, et dont il n'est pas toujours facile de suivre le fil conducteur. L'habitation No 7 avait jadis été attribuée définitivement aux Acadiens Paul Boudrot et sa femme Françoise Daigle par la transaction du 14 octobre $1791^{25}$. Le 27 septembre 1792, Boudrot meurt. L'épouse et les enfants héritent de la totalité des biens, qui sont partagés le 19 floréal an X. Le No 7, estimé, terres et bâtiments, à la modique somme de $522 \mathrm{Fr}$. 24, passe aux mains d'un fils, Benjamin Boudrot ${ }^{26}$.

C'est peu après que se produit cette évolution si souvent consta-

24. Minutes Vézien.

25. Minutes Pleignard.

26. Minutes Amirault. 
tée pour les jeunes métairies acadiennes, à savoir le glissement de l'exploitation vers une vieille métairie avoisinante, qui attire dans son orbe les terres dont le défrichement est parfois inachevé. Le No 7 est d'abord vendu par Benjamin Boudrot à son beau-frère Thomas Gervais, maire de La Puye, pour 1800 francs $^{27}$. Gervais, qui semble avoir soigneusement préparé son affaire, revend sept jours après, le 28 thermidor, an XI, son acquisition à un bourgeois rural d'Archigny, Beaudet des Roches demeurant à Joline, à quelque deux kilomètres de là̀ ${ }^{28}$.

La famille Beaudet des Roches, ainsi introduite dans le circuit acadien, connaît par la suite de nombreux deuils. Remariages et bouleversements familiaux font qu'en 1819 la jeune Marie-Anne Beaudet, orpheline et mineure, a besoin d'un tuteur. On lui en donnera un en la personne de Bernard Texier.

Nous n'avons point d'exemple, sous la Révolution et l'Empire, de tuteur qui se soit jamais ruiné en gérant les affaires de sa pupille. Lorsque madame Carré de Busserolles, restée seule avec quatre enfants après l'émigration de son mari, mourut dans la prison de Châtellerault, sous la Terreur, il fallut bien trouver un tuteur aux orphelins. C'est le notaire Drouault qui fut chargé de cette mission de confiance ${ }^{29}$. Il n'y perdit point. Texier n'était pas plus apparenté aux Beaudet des Roches que Frouault aux Busserolles. On le nomma tuteur en raison de sa compétence aux affaires et de son activité. Le 30 août 1819, il fit procéder à l'inventaire des meubles et effets revenant à sa pupille et se montant à la coquette somme de $7748 \mathrm{f}$., $65^{30}$. Le 18 mars 1821, on le voit encore s'occuper du patrimoine des Beaudet ${ }^{31}$. Nous ne regretterons jamais assez de ne pas connaitre les archives de familles qui nous permettraient sans doute de prouver ce que nous soupçonnons déjà: l'enrichissement accéléré du ménage Texier, parallèlement à l'appauvrissement de l'autre ménage, celui de Françoise Reine, fille aînée de l'Acadienne Marie-Reine Berbudeau.
27. Ibid.
28. Ibid.
29. Archives de la Vienne. $Q^{2} 43$.
30. Minutes Collet.
31. Ibid. 
C'est à la même époque, en 1819, que Texier devint percepteur de Bonneuil-Matours, fonctions pour lesquelles il doit verser un cautionnement de 1034 francs $^{32}$. En 1826, il est membre du conseil de fabrique, chargé de gérer les affaires de la paroisse ${ }^{33}$. Collecter les contributions n'a jamais été un travail de tout repos. Le rôle de fabricien ou fabriqueur, comme on dit à l'époque, revêt un caractère moins absorbant. Il apparaît comme une consécration sociale souvent recherchée, et achève de ranger le fils de l'huilier dans les personnalités locales de premier plan.

\section{III}

Époux d'une fille d'Acadienne, voici que le destin le ramène à nouveau sur la Ligne, comme il avait ramené son beau-frère, l'infortuné boulanger Bruneau. Ces maisons que les réfugiés du Grand Dérangement avaient trouvées environnées de brandes, qui virent les dernières années du grand-père Berbudeau, l'ancien subdélégué de l'Ile Saint-Jean, exercent encore, un quart de siècle après, une singulière attraction sur le jeune marchand enrichi.

Lors du partage, que nous avons évoqué tout à l'heure, des biens de Paul Boudrot et Françoise Daigle, le 19 floréal an X, une métairie acadienne, le No 16, était échue à Paul Boudrot fils. Celuici ne la conserva pas plus que son frère Benjamin avait gardé le No 7. Il commença par l'affermer, le 18 ventôse an XII, pour sept ans moyennant 60 francs annuels, au même Thomas Gervais qui avait acheté le No $7^{34}$. Puis, sans même attendre la fin du bail, il l'échangea, le 25 floréal an XII, à Jean Ploudre, cultivateur, contre 19 pièces de terres éparpillées en labours, prés, et chenevières, dans la commune de Cenant.

Des 174 hectares que comprenait à l'origine le No 16 , il ne restait plus que 2 hec. 40 de terres labourables et 8 hectares de brandes ${ }^{35}$.

32. Archives de la Vienne. S P Ch. 110, No 3266.

33. Registre de fabrique de Bonneuil-Matours. Ce très intéressant document nous a été fort obligeamment communiqué par M. l'abbé Sardet. Il commence en 1787 et fut, depuis cette date, utilisé sans interruption par les curés de BonneuilMatours, comme il l'est encore de nos jours.

34. Minutes Amirault.

35. Minutes Collet. 
Démembrement que Pérusse d'Escars, en légiférant pour l'installation de la colonie, n'avait sûrement point prévu. Le 13 juillet 1829 , il nous est donné de visiter en détails cette maison acadienne, habitée désormais par des laboureurs du pays.

Jean Plourde vient de mourir. Ses héritiers, au nombre de six, y compris la veuve, demandent un inventaire. Pénétrons, à la suite du notaire Collet et de deux voisins choisis comme experts, dans le logis où nous constatons que, des deux pièces habitables, une seule est utilisée. On y entasse, dans l'étroit espace dont nous connaissons les dimensions ${ }^{36}$, trois lits à quenouilles, trois coffres, un buffet, une armoire à deux battants, un vaisselier, une maie, une table ronde et ses quatre chaises, un rouet avec ses fuseaux. Il ne reste plus grande place pour se mouvoir. L'autre pièce sert de débarras. Le cellier et la grange possèdent différents objets et outils de travail. Dans l'écurie, deux mauvaises juments. Les bœufs de labour que contient invariablement, à cette époque, toute métairie de rang moyen, sont absents. Tous détails qui nous permettent de définir le No 16, en 1829, comme une borderie de peu d'envergure. Depuis son échange de l'an XII, Plourde en a seulement porté la surface de 10 hec. 40 à 12 hectares. Ajoutons qu'elle contient toujours des brandes dont nous ignorons l'étendue, ce qui nous permet de dire que le défrichement n'a pas été poussé jusqu'au bout.

Sortie du périple acadien par l'échange du 25 floréal an XII, le No 16 va y retourner en passant sous la coupe d'un Po tevin devenu, par son mariage, petit-fils d'Acadien. Mais l'opération n'est pas simple. D'après un testament fait par Plourde le 12 décembre 1828 , chaque enfant du défunt possède, d'une part la sixième partie indivise de l'exploitation, d'autre part la cinquième partie également indivise d'un autre sixième dont la veuve a la jouissance en usufruit pendant sa vie. Tels sont les faits. Ces fractions de fractions sont fusionnées par Bernard Texier, qui le 6 décembre 1829, ne s'attardant pas à de telles subtilités arithmétiques, met d'accord cinq héritiers sur six, moyennant 1000 francs, à savoir 200 francs pour chacun des 4 enfants consentants et autant pour les droits de jouissance de la veuve.

Le nœud gordien tranché, il restait encore à convaincre Jean

36. Gónéral Papuchon, La Colonie acadienne du Poitou (Poitiers, 1908), 49. 
Denis, journalier à La Puye, époux d'une fille Plourde, qui s'entêtait à maintenir un droit de propriété aussi dérisoire qu'inapplicable sur une partie du No 16. Il se décida, le 18 avril 1830, à capituler. Mais, s'il avait pensé tenir la dragée haute à son acheteur en le faisant attendre le dernier lambeau du domaine acadien, il commit une faute de psychologie. Sa portion fut payée exactement le même prix que les autres, c'est-à-dire 200 francs $^{37}$.

Qu'advint-il de cette maison? Demeura-t-elle longtemps dans le patrimoine des Texier? Le nouveau propriétaire lui fit-il subir des améliorations, des agrandissements? Nous n'avons encore pu avoir accès aux documents nous permettant de répondre à ces questions. La fille cadette de Marie-Reine Berbudeau et son mari échappent à nos recherches après l'achat du No 16. Quelques dates et points de repère permettent seuls d'affirmer que le percepteur de Bonneuil-Matours paracheva son élévation sociale au cours de la monarchie de Juillet.

En 1832, de marchand et fermier, il est devenu propriétaire et adjoint au maire de Bonneuil-Matours. En 1839, il est maire luimême $^{38}$ comme son beau-père, le syndic des Acadiens, l'avait été en 1793 à Archigny. Il le restera longtemps, jusqu'à 80 ans, en 1862, date de sa mort. Depuis 1850, Marie-Anne repose dans le nouveau cimetière, car en 1834, on a transféré l'enclos des trépassés dans un lieu plus éloigné du bourg ${ }^{39}$. Agrandi, le champ de foire a recouvert l'emplacement de l'antique nécropole paroissiale où Augustin Texier, le gagne-petit, allait semer, autour des murs, une luzerne éphémère. Pareillement, l'oubli a recouvert dans le pays le souvenir de Bernard Texier, maire de Louis-Philippe, de la Seconde République, conscrit défaillant de l'an XI. Retenons qu'il offre avec sa femme, fille d'Acadienne, l'image complète de la réussite. Du bonheur? Ce mot ne figure pas dans le vocabulaire des historiens.

Pierre Massé, Paris, (France)

37. Minutes Collet.

38. Reg. de délib. de la municipalité de Bonneuil-Matours.

39. Archives Nationales. $F^{2}$ II Vienne 3; Archives de la Vienne. S P Ch. 95. 